Revue des patrimoines

$37 \mid 2018$

Jardins collectifs : de l'abbé Lemire aux jardins d'insertion. Typologies - Expériences - Enjeux de conservation

\title{
Des jardins familiaux centenaires labellisés : quel avenir ? L'exemple du site Paul Philippe à
} Versailles

The Paul Philippe gardens at Versailles, one-hundred-year-old allotment

gardens awarded a special label, but what is their future?

\section{Pierre Desnos}

\section{(2) OpenEdition}

\section{Journals}

Édition électronique

URL : http://journals.openedition.org/insitu/18798

DOI : 10.4000/insitu. 18798

ISSN : 1630-7305

Éditeur

Ministère de la culture

Référence électronique

Pierre Desnos, «Des jardins familiaux centenaires labellisés : quel avenir ? L'exemple du site Paul Philippe à Versailles », In Situ [En ligne], 37 | 2018, mis en ligne le 12 décembre 2018, consulté le 19 avril 2019. URL : http://journals.openedition.org/insitu/18798 ; DOI : 10.4000/insitu.18798

Ce document a été généré automatiquement le 19 avril 2019

\section{(c) $(1) \odot$}

In Situ Revues des patrimoines est mis à disposition selon les termes de la licence Creative Commons Attribution - Pas d'Utilisation Commerciale - Pas de Modification 4.0 International. 


\section{Des jardins familiaux centenaires labellisés : quel avenir ? L'exemple du site Paul Philippe à Versailles}

The Paul Philippe gardens at Versailles, one-hundred-year-old allotment gardens awarded a special label, but what is their future?

Pierre Desnos

\section{De la labellisation et de ses effets}

$1 \mathrm{Si}$, pour les spécialistes des jardins collectifs, la distinction peut sembler superfétatoire, c'est loin encore d'être une évidence pour de nombreux responsables et élus actuels ${ }^{1}$. Et confondre les jardins familiaux avec les jardins partagés n'aide pas à protéger les premiers qui font partie du patrimoine ni à promouvoir les seconds qui constituent l'expression actuelle - et peut-être future - des jardins collectifs. Si les jardins familiaux du début $\mathrm{du} \mathrm{XXI}^{\mathrm{e}}$ siècle sont bien les héritiers des jardins ouvriers de la fin du XIX ${ }^{\mathrm{e}}$ siècle, ils en diffèrent fortement par leur diversité : historiques ou récents, urbains ou ruraux, en association loi 1901 ou en association adossée à une commune, purement familiaux ou à coloration partagée. Cette diversité rend complexe la question de leur conservation et de leur protection, qui sont pourtant nécessaires, à moins de considérer qu'étant immatérielles, les traces de l'histoire sociale d'une ville ou d'une région n'ont pas la valeur des témoins historiques construits.

2 Labelliser pour protéger, certes, car toute reconnaissance contribue à préserver. L'expérience récente montre pourtant que cela ne saurait suffire et qu'il faut légitimement se poser la question de l'avenir des jardins familiaux "pur jus", historiques, urbains, pleinement autonomes et « labellisés » comme ceux de Versailles. Et le danger ne vient pas toujours de là où on l'attend. En effet, le label «Jardin remarquable » accordé en décembre 2014 au site Paul Philippe de Petits-Bois géré par l'Association des jardins familiaux de Versailles et des communes environnantes s'est 
avéré à double facette : d'un côté la reconnaissance semble avoir valu relative protection, d'un autre côté la labellisation et ses exigences ont posé avec acuité la question de la gestion de ces jardins (brochure à réaliser, ouverture du site avec la problématique de la compétence et de la disponibilité de jardiniers pour accueillir les visiteurs, obligation d'amélioration de l'aspect esthétique du site tout en conservant la diversité et le caractère non policé des jardins familiaux, acquisition et transmission de savoirs de gestion).

Du fait du développement urbain, les jardins familiaux susceptibles d'être labellisés ou protégés au titre du patrimoine historique se situent pour l'essentiel au cœur des villes, ou pour le moins dans des périphéries fortement urbanisées, et sont donc devenus au fil $\mathrm{du}$ temps des jardins familiaux urbains dont la gestion (administrative, financière, humaine et environnementale) diffère notablement de celle des jardins familiaux ruraux. Par son statut indirectement protecteur, la labellisation d'un site doit tenir compte de cette réalité et l'accompagner.

\section{Pour durer, il faut une gestion pérenne}

4 Si l'on veut conserver des sites de jardins familiaux, il faut bien entendu en connaître l'histoire et l'importance locale, mais si l'on veut aussi les accompagner vers l'avenir il faut surtout en comprendre le mode de fonctionnement. Les associations de jardins familiaux sont gérées par des bénévoles qui, en cas de labellisation ou de protection, sont confrontés à des problèmes nouveaux pour lesquels ils ne sont généralement pas armés. La labellisation ou la protection considère injustement qu'elle suffit à préserver les sites remarquables qu'elle reconnaît. Pour être complète et cohérente, elle se doit pourtant de prévoir des outils qui en facilitent et améliorent la gestion, pierre angulaire de la pérennité. En observant l'histoire de l'Association des jardins familiaux de Versailles et des communes environnantes, cet article montre comment l'évolution de cette institution a été rythmée par les conditions dans lesquelles s'est exercée sa gestion.

5 Si l'on considère l'histoire de cette association en termes de gestion, ses cent quinze années d'existence se subdivisent en trois périodes: les philanthropes (une trentaine d'années), les élus (une cinquantaine d'années) puis les jardiniers (une trentaine d'années). Cet article parcourt rapidement les deux premières périodes et s'attarde sur la troisième en recueillant le point de vue des administrateurs actuels sur les conséquences de la labellisation du site Paul Philippe et sur les attentes qu'elle a fait naître.

\section{1-1931. Les philanthropes : des gestionnaires avisés}

6 L'CEuvre des jardins ouvriers de Versailles est créée en mai 1901 grâce à une généreuse bienfaitrice qui charge quelques notables versaillais d'employer «à une œuvre populaire utile une somme de 3500 francs ». Après avoir constaté la viabilité de leur œuvre, les premiers philanthropes transforment en 1903 leur première association en une seconde, rapidement renommée Société des jardins ouvriers de Versailles et des communes environnantes, la transformation visant à élargir le rayon d'action de ladite société. Parmi les notables qui s'engagent dans l'administration de l'œuvre, nous trouvons un rentier (M. Berga), un maraîcher (M. Poirier), un baron (M. de Schonen), un abbé 
(M. Boyer) ${ }^{2}$, un lieutenant-colonel en retraite (M. Janin), un architecte (M. Blondel), un docteur en droit (M. Galinier) ou encore un notaire (M. Conrad Munsch). Par leur position sociale et leur formation, ces notables connaissent tous les rouages de la société et savent saisir toutes les opportunités financières de l'époque. Ainsi, ils sauront s'attirer les subventions, négocier le coût de l'eau, utiliser des prêts comme les fonds du Pari mutuel, et surtout investir habilement pour acquérir le terrain de Petits-Bois. Pour acheter en 1914 ce terrain de 1,89 ha loué depuis 1906, neuf philanthropes administrateurs se constituent bailleurs de fonds d'un prêt hypothécaire portant intérêt de $4 \%$ et remboursables en 28 ans. Le montage financier s'effectue sans recourir aux banques et prévoit un paiement par subrogation, c'est-à-dire que les neuf bailleurs demeurent solidairement les propriétaires du bien acquis tant qu'ils ne sont pas intégralement remboursés. La Société des jardins ouvriers ne possède donc son terrain qu'à l'extinction de la dette qui intervient plus tôt que prévu grâce à la vente d'une partie dudit terrain, à une gestion financière rigoureuse, à l'augmentation des redevances des tenanciers (c'està-dire des jardiniers ouvriers) et à la multiplication des subventions. À titre d'exemple, les redevances des tenanciers s'élevaient en 1907 à $88,10 \mathrm{~F}$ et les subventions à $36 \mathrm{~F}$ pour des recettes de $4455,55 \mathrm{~F}$, ces contributions respectives étaient en 1923 de $6423,90 \mathrm{~F}$ et $2500 \mathrm{~F}$ pour des recettes de 11 856,55 F (fig. 1). La Société des jardins ouvriers devient définitivement propriétaire de son terrain de Petits-Bois et des constructions attenantes en 1929, cette possession en propre garantissant la pérennité du site en tant que jardins familiaux.

Figure 1

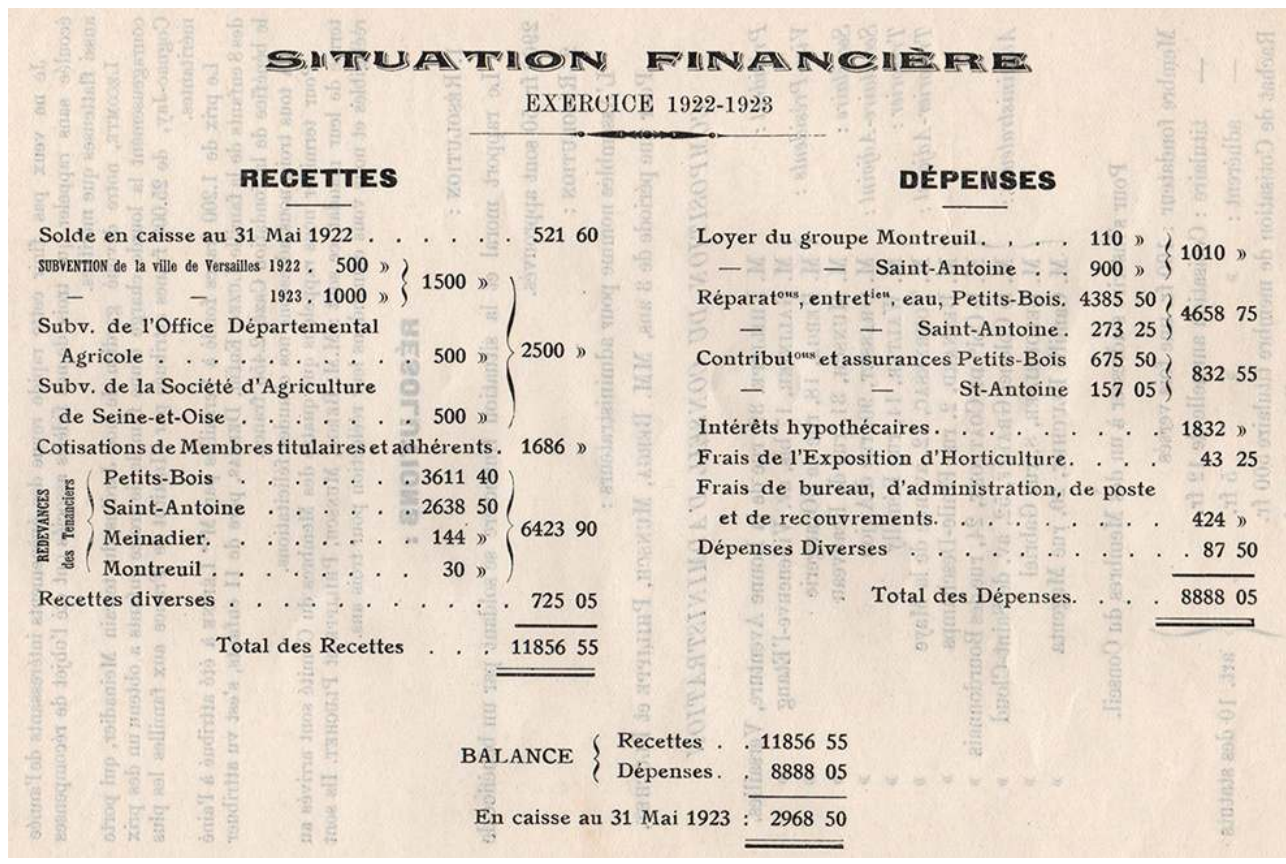

Page du résultat comptable de l'Association des jardins ouvriers de Versailles présenté à l'assemblée générale du 17 juin 1923 - Rapport imprimé du conseil d'administration - Situation financière exercice 1922-1923. Archives communales de Versailles 6 Z $6^{*}-8^{*}\left(f^{\circ} 163\right)$

(c) Archives communales de Versailles.

7 En termes de pratiques culturales et d'environnement, les trente premières années des jardins ouvriers à Petits-Bois se distinguent par une totale liberté dans la nature des cultures (une seule exigence : un jardin mixte), dans l'aménagement des parcelles (« abris 
de fortune ", clôtures anarchiques) et dans le comportement (l'alcoolisme étant à peu près le seul tabou). Dès les origines, la discipline est assurée par le gardien engagé et logé par l'association, une situation qui préserve en grande partie les gestionnaires des épineuses questions relationnelles et des inévitables conflits du quotidien. Au bout du compte, les philanthropes auront été des gestionnaires prévoyants et avisés.

\section{2-1985. Les élus : des gestionnaires opportunistes}

Dès le début des années 1930, des élus de Versailles et des communes environnantes prennent place au sein du conseil d'administration. La présidence de l'association est ainsi assurée par Conrad Munsch, maire-adjoint de Versailles, de 1932 à 1942, par André Crozet, maire du Chesnay, de 1943 à 1948, puis par Émile Fuzellier, maire-adjoint de Versailles, de 1948 à 1976. La dernière présidence par un élu est assumée de 1976 à 1984 par Philippe Capelle, conseiller municipal du Chesnay. La trésorerie est prise en charge de 1948 à 1979 par un directeur de banque, F. Gilbert. Le positionnement de ces administrateurs dans les processus de décision de la vie locale et économique leur permet de saisir aisément les opportunités d'expansion en période de guerre puis de résister efficacement à la pression foncière pendant les Trente Glorieuses. À titre d'exemple, profitant des lois réquisitionnaires du 18 et du 27 août 1940 qui ont favorisé la création de jardins de guerre sur les terrains abandonnés ou incultes, les administrateurs de l'Association des jardins ouvriers de Versailles vont étendre notablement leur territoire et le nombre de leurs jardins en exploitation, qui atteint 1464 jardins sur 25 hectares en 1943.

9 Si l'après-guerre se révèle dans un premier temps difficile à gérer en raison de la restitution des terrains réquisitionnés mais aussi de la révolution urbaine qui s'engage et menace les jardins ouvriers "rurbains", les administrateurs sauront y faire face efficacement. Les premières attaques pour le site de Petits-Bois se manifestent dès 1955, lorsque le maire de Versailles signifie à l'association « qu'il est indispensable d'envisager pour l'avenir le déplacement des jardins ouvriers qui ne pourront pas demeurer dans un quartier à bâtir... $»^{3}$. Le refus du conseil d'administration et les protestations des jardiniers ont certainement contribué à sauvegarder le site mais on peut aussi raisonnablement penser que la balance a penché du bon côté parce que le président des jardiniers familiaux était l'adjoint au maire. À partir des années 1960, face à la poussée immobilière et à la perte d'intérêt pour la culture en milieu urbain, l'association perd la plupart de ses sites en location (127 jardins au Chesnay en 1962, 130 jardins à Rocquencourt en 1971) et cède du terrain sur le site de Petits-Bois. Mais chaque cession sur le terrain versaillais enrichit financièrement l'association, qui peut même envisager en 1966 d'acheter de nouveaux terrains, ce qu'elle ne fera pas, se contentant de thésauriser (fig. 2). 
Figure 2

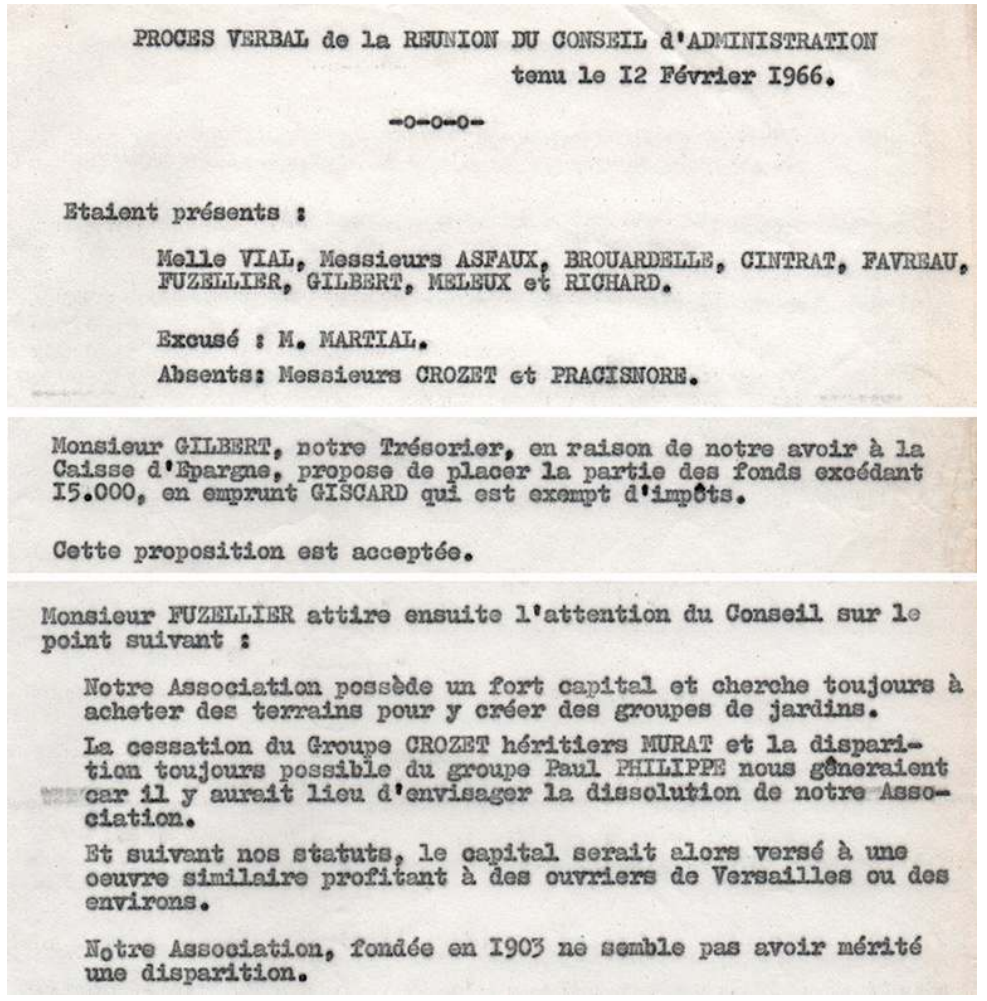

Extraits du procès-verbal du conseil d'administration de l'Association des jardins ouvriers de Versailles du 12 février 1966.

(c) Archives de l'Association des jardins familiaux de Versailles.

En termes de pratiques culturales et d'environnement, les cinquante-deux années des jardins ouvriers et familiaux à Petits-Bois gérées par les élus ne se distingueront guère des trente premières années, à une différence notable près : l'obligation de s'adapter à l'urbanisation de l'environnement. Ainsi, les administrateurs prennent conscience en 1971 « du mauvais état des abris, ce qui donne un aspect de «zone» à nos jardins et ne plaide pas en faveur de leur maintien » et décident que les abris de jardins seront dès lors achetés et gérés par l'association. Les cultures suivent quant à elles l'air du temps en recourant généreusement aux intrants chimiques, la taille des légumes continuant à l'emporter sur l'esthétique des jardins. Comme les philanthropes, les élus se reposent sur le gardien pour assurer la discipline et la gestion du quotidien. Au final, les élus gestionnaires auront su prudemment traverser les turbulences des Trente Glorieuses en faisant preuve parfois d'un opportunisme de bon aloi.

Comme nous pouvons le voir, les quatre-vingts premières années de l'Association des jardins ouvriers et familiaux de Versailles se sont caractérisées par des équipes d'administrateurs bien au fait de la gestion, de la finance et des questions administratives, autant de compétences qui, alliées à un fort entregent local, ont participé à la vitalité et à la pérennité de l'institution mais aussi à la préservation du site Paul Philippe de Petits-Bois. 


\section{6-2017. Les jardiniers : des gestionnaires contraints}

12 En 1986, tous les élus et notables sont partis. Les jardiniers prennent leur destinée en main... La gestion avant ce passage de témoin sera longtemps un sujet d'interrogation de la part des nouveaux administrateurs. En 2011, certains anciens jardiniers laissent encore poindre quelque ressentiment. Le livre comptable de l'association consignant les exercices de 1954 à 1986 ne nous éclaire pas sur cette question. On peut cependant s'interroger. On y relève en effet qu'au 31 décembre 1978, le trésorier de l'époque, M. Gilbert, fait état d'une réserve "foncière » s'élevant à 745144,52 francs après prélèvement des 381584,15 francs pour les abris de jardin et le ravalement de la maison du gardien. Au 31 décembre 1979, cette réserve foncière n'apparaît plus dans le livre comptable... Les jardiniers en deviendront méfiants. À partir de 1987, l'Association ne bénéficie plus d'aucune subvention'.

La présidence de l'association est assurée de 1986 à 2002 par Jean-Jacques Lepage, directeur de l'école primaire Yves Le Coz à Versailles, avec un intermède de quatre années, entre 1996 et 2000, où cette fonction revient à un autre jardinier, Philippe Robin. C'est l'heure du repli sur soi de l'association: plus d'adhésion à la Ligue du coin de terre, disparition des subventions et finances à plat. La rigueur gestionnaire s'avère impossible pour des jardiniers bénévoles, sans compétences nécessaires pour administrer une association disposant d'un bien immobilier. Souvent exercée dans l'urgence, la gestion devient approximative et sujette à caution. Ainsi, le poste de trésorier est-il confié pendant près de trois décennies au gardien qui, tout en étant jardinier, bénéficie à titre gracieux du logement dans la maison appartenant à l'association, avec en seule contrepartie l'obligation d'assurer la discipline dans les jardins et de menues tâches.

\section{7-2014. Des événements révélateurs pour les jardiniers gestionnaires}

14 Si les compétences juridiques, administratives et financières s'amenuisent, la question de la « bonne gestion » ne se pose guère entre 1985 et 2007, la période s'avérant plutôt calme pour les jardins familiaux. Un projet foncier engagé par l'O.P.H. (Office public de l'habitat) Versailles Habitat change la donne en 2007 car une partie du terrain et la maison des jardins familiaux sont destinées à être absorbées par le projet immobilier dénommé « La porte des jardins ». Les jardiniers administrateurs s'interrogent tout à coup sur leur statut, sur leur avenir et sur leur capacité à résister. Après avoir accepté le projet, ils se tournent vers l'extérieur en 2010. L'ouverture de leurs archives permet à l'auteur du présent article d'écrire l'histoire de ces jardins (2011) ; le livre conduira quant à lui à une reconnaissance par la municipalité (2012) qui initie à son tour une démarche de protection, laquelle aboutit à la labellisation « Jardin remarquable » en 2014. On aurait pu s'attendre à ce que cette reconnaissance aide l'association à mieux négocier les changements générés par le projet immobilier (disparition du local associatif et du logement du gardien, gestion d'un logement de ville hérité du projet, suppression du poste de gardien, nouvelles règles de discipline, changement de statut administratif et fiscal). Il n'en fut rien. La labellisation a apporté au contraire un surplus de charges pour 
les administrateurs: rédiger et publier une brochure de présentation du «jardin remarquable ", ouvrir au public pendant plusieurs week-ends dans l'année, convaincre les farouches jardiniers familiaux de se montrer accueillants après avoir vécu longtemps à l'abri des regards, soigner l'image du site en respectant la nature de l'activité ou encore faire évoluer les pratiques environnementales (récupération des eaux pluviales, zéro phyto et compostage).

Pour toutes ces tâches, l'association ne reçoit pas ou peu de soutien. La fondation Georges Truffaut verse bien 100 euros (!) pour aider les jardins familiaux de Versailles (dans le même temps, elle sponsorise à hauteur de 3000 euros la création d'un jardin partagé distant de $300 \mathrm{~m}$ ). La Ville de Versailles consent à financer l'impression de la brochure grâce à l'appui du maire, François de Mazières, soucieux de soigner l'image de sa « ville verte ». Au-delà de ces deux aides somme toute modestes, les administrateurs ont dû faire face seuls. La fragilité de l'institution reposant sur quelques épaules est alors pleinement ressortie. Le faible renouvellement des gestionnaires est devenu problématique et les quelques administrateurs impliqués se lassent ou s'épuisent face aux difficultés de la gestion quotidienne pour lesquelles il n'existe que des réponses parcellaires : la juridique (que faire par exemple en présence d'un jardinier exclu qui refuse de quitter sa parcelle? ), la fiscale (comment s'acquitter de ses obligations face à une administration qui ne connaît pas tous les droits des jardins familiaux ?), l'économique (comment gérer un bien immobilier dont on ne peut pas utiliser de façon pérenne les fonds produits?), l'humaine (comment assurer la discipline dans les jardins sans s'exposer personnellement, tout en demeurant un jardinier?).

16 La labellisation du site Paul Philippe de Petits-Bois a ainsi confronté les administrateurs des jardins familiaux à des problématiques nouvelles : ouverture vers l'extérieur, remise en cause de pratiques anciennes, découverte des différences entre jardins familiaux urbains et ruraux, complexité d'une gestion administrative difficilement maîtrisable par des bénévoles eux-mêmes jardiniers et disposant rarement des connaissances pour affronter les arcanes des administrations.

17 - Témoignage de Marcel Saintonge, 62 ans, retraité PSA depuis 2015 et président de l'Association des jardins familiaux de Versailles depuis 2002 :

Pour moi, en tant que président de l'association, la labellisation a beaucoup changé mon investissement de temps, beaucoup de contacts avec les élus, les institutionnels, la presse et la radio, beaucoup de nouveaux critères à respecter dont les 52 jours d'ouverture au public qui nous ont obligés à trouver une école pour valider ces journées, beaucoup de dossiers à remplir pour les différentes journées, il y a surtout ces cinq week-ends obligatoires qu'il a fallu organiser et où il a fallu être présent, beaucoup de temps, beaucoup de temps. Avec la gestion courante qui est tout sauf simple, j'en suis presque arrivé à une activité à plein temps, du cent pour cent pour remettre à jour les jardins, pour les rendre plus présentables, plus beaux et faire respecter nos nouvelles obligations vis-à-vis de l'extérieur. La labellisation nous a obligés à faire des améliorations comme notre plaque d'entrée, il serait bon que les pouvoirs publics ou la mairie prévoient quant à eux une signalétique pour mieux localiser le site, et pourquoi pas aussi un panneau historique présentant le site comme cela se fait beaucoup dans Versailles. L'implication de la reconnaissance ne reviendrait ainsi pas aux seuls jardiniers. Nous nous sentirions moins seuls.

18 - Témoignage de Mathieu Laurent, 31 ans, agent de conduite à la SNCF, trésorier de l'Association depuis 2015 : 
Quand je suis devenu trésorier, la labellisation venait d'être mise en place. J'ai tout de suite vu qu'elle nous générait de nouveaux postes de dépense sans qu'il y ait de rentrées qui compensent. Heureusement, l'impression de la plaquette de présentation de notre site Paul Philippe avait été prise en charge par la mairie de Versailles, il est vrai après un gros forcing de notre vice-président auprès du maire. Après, il faut bien avouer que la labellisation peut coûter cher car elle oblige tout de même à tenir le site propre et bien entretenu, ce qui veut dire aussi préserver l'authenticité du site en utilisant des matériaux qui ne sont pas toujours faciles à trouver aujourd'hui et qui sont donc plus coûteux qu'à l'époque où ils ont été mis en place. Pour Paul Philippe, je pense en particulier aux bacs à eau en béton qui ont près de cent ans mais aussi au réseau d'alimentation en eau qui est vétuste et hétéroclite. Cette partie financière n'est évidemment pas négligeable. Après, il y a la question de l'ouverture au public pour laquelle il faut prévoir de l'information, nous avons réalisé pour cela quatre bâches plastiques imprimées qui n'ont pas été gratuites. Et puis pour nous labellisation a voulu dire également la création d'un site internet pour offrir une bonne communication et une meilleure visibilité, aujourd'hui c'est indispensable, ça non plus n'a pas été gratuit. Sans la labellisation, il n'y avait pas vraiment de nécessité.

\section{Pour une meilleure reconnaissance du bénévolat}

Pour contribuer à la pérennisation des jardins familiaux, il faut encourager l'engagement des jardiniers dans la vie de leur association afin d'assurer une relève des gestionnaires en assurant la formation appropriée des nouveaux. Ceux-ci étant bénévoles, la lassitude s'installe au fil des ans d'autant plus que leur travail n'est pas toujours reconnu à sa juste mesure, en particulier les heures consacrées au simple bon fonctionnement de l'association. D'après les observations de l'auteur, qui assume la fonction de viceprésident depuis trois années, le cumul des heures consacrées à la gestion par le président et le trésorier équivaut probablement à un emploi à temps plein sur une année pour une personne... un emploi à temps plein ni rémunéré, ni même indemnisé, la loi considérant toute gratification d'un bénévole comme un salaire ou un avantage en nature.

La création en 2017 d'un nouveau site de jardins a mis en évidence le caractère pernicieux d'un bénévolat où toute indemnisation est exclue. Il est en effet difficile de dire à un jardinier en activité professionnelle que les dizaines d'heures consacrées à aménager un site dont il ne bénéficiera pas seront sans gratification autre qu'un « merci » (fig. 3). Pour encourager l'investissement bénévole des jardiniers, il serait judicieux de prévoir une indemnisation des bénévoles en fixant un plafond suffisamment bas pour ne pas entrer dans la sphère du travail rémunéré mais permettant au moins de fidéliser l'engagement continu et effectif au sein de l'association, en particulier lorsque celle-ci dégage une marge bénéficiaire, sans pénaliser les adhérents. L'indemnisation devrait évidemment être définie et votée lors de l'assemblée générale annuelle de l'association. Pour cela, il faut revisiter la loi de 1901, une approche que d'aucuns ont déjà envisagée pour favoriser la vie associative et encourager l'engagement citoyen. 


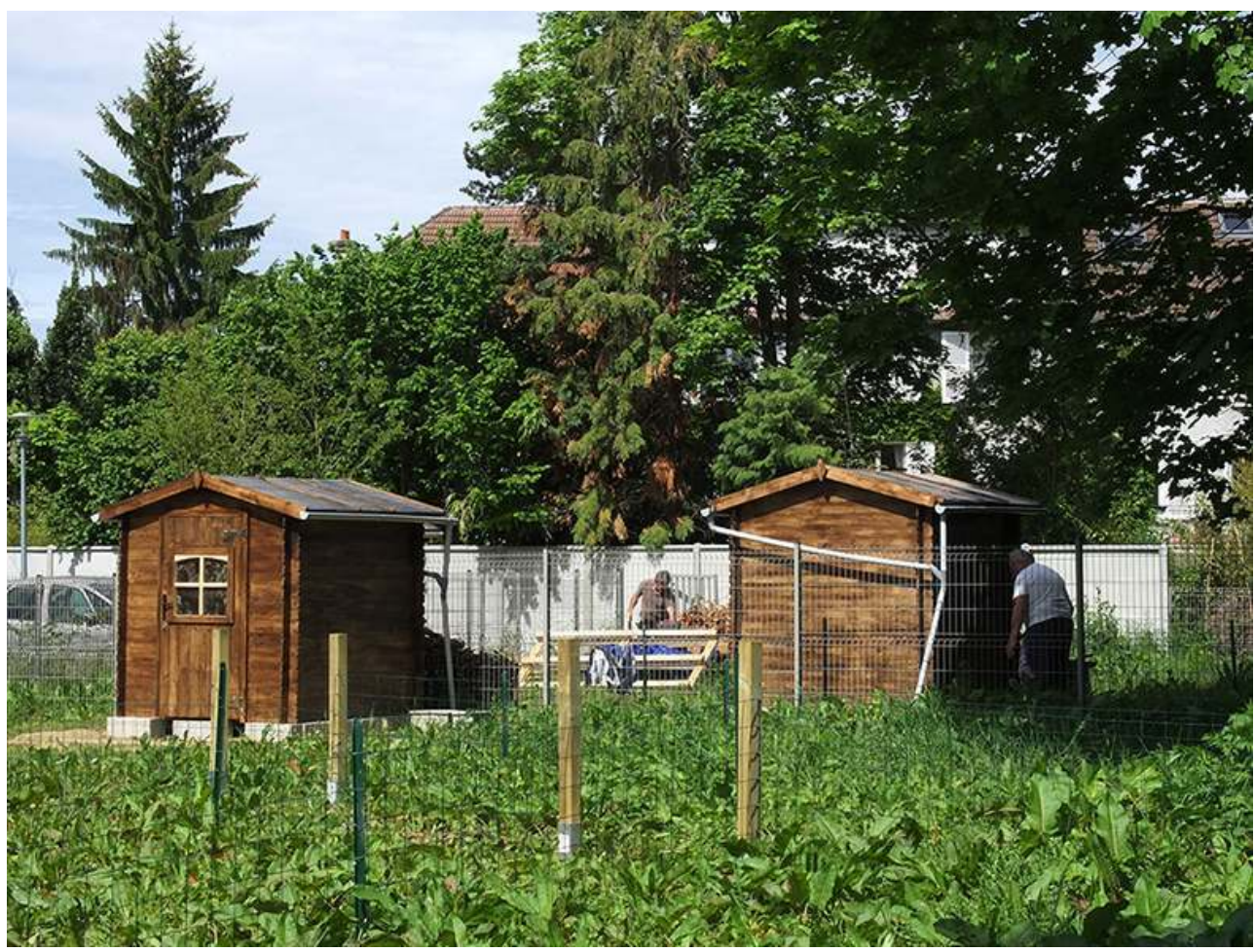

Création du site urbain de 8 jardins familiaux rue de l'Ermitage à Versailles (cabanes subventionnées par Versailles Habitat, 19 jardiniers bénévoles participant à l'aménagement, 440 heures-homme de travail, coût pour l'association environ 6000 euros).

(c) Association des jardins familiaux de Versailles.

21 Lorsque l'on pose la question de l'aide et du soutien que les jardins familiaux pourraient attendre dans le prolongement d'une labellisation ou d'une protection, voici ce qu'en pensent les principaux responsables de l'association:

- Marcel Saintonge, président de l'Association des jardins familiaux de Versailles :

En ce qui concerne l'aide proprement dite que nous pourrions attendre des instances qui nous protègent par la labellisation, je pense qu'il faudrait qu'un intervenant extérieur ayant fait des études d'histoire puisse venir nous aider à présenter des informations solides, car si notre vice-président historien n'avait pas été là nous aurions quand même eu pas mal de difficultés à faire des visites intéressantes pour les Journées du patrimoine. Alors oui, il serait souhaitable qu'une aide de sachants nous soit détachée lors des journées officielles d'ouverture des jardins : des historiens évidemment, mais aussi des botanistes car il faut bien dire que les jardiniers ne connaissent pas toujours les noms et les caractéristiques des fleurs et des légumes qu'il y a dans leur jardin... et quand on sait qu'il y a plus de 200 espèces et 580 variétés de plantes sur le site Paul Philippe, je vois mal le commun des jardiniers familiaux ne pas dire de bêtises. Même dans le bureau, il n'y en a pas beaucoup qui savent parler en public ou qui sauraient quoi dire. Sachant que l'on a déjà du mal à trouver six personnes sur quatre-vingts jardiniers pour siéger au conseil d'administration, on mesure le chemin à parcourir pour maîtriser cette question des visites. Bon, c'est vrai que la labellisation est un changement à 180 degrés. Il nous faudrait plus de volontés, plus de disponibilités et plus de compétences de la part des jardiniers, qui sont tout de même des solitaires.

- Mathieu Laurent, trésorier de l'Association : 
Pour ce qui est de l'aide financière, certains projets mériteraient une aide extérieure. Sur le site Paul Philippe, il y a bien sûr la restauration du circuit d'alimentation en eau, ce gros projet que nous avons soumis à l'Agence de l'eau a reçu une réponse négative pour des subventions en dépit des avancées écologiques que nous avions proposées comme la récupération des eaux pluviales sur les bâtiments alentour et les bâches de rétention permettant de réduire la consommation d'eau de ville. Ce gros projet est donc bloqué car c'est un investissement énorme et nous ne pouvons pas augmenter les cotisations qui, avec 80 euros, sont parmi les plus basses de France. Nous voulons garder notre caractère social. Là, nous pourrions espérer un soutien des instances qui labellisent. Ce serait bien aussi de recevoir une aide dans les démarches administratives de tous types car la paperasse n'est pas toujours évidente, un ou deux jours par an au départ cela aiderait bien, car la labellisation c'est vraiment un surplus de travail pour les bénévoles que nous sommes.

24 Les jardins familiaux sont des espaces privés dans un espace privé : des parcelles louées à des jardiniers dans un site géré par une association loi 1901 qui le possède ou l'occupe à titre gratuit ou payant. Si le site de jardins familiaux susceptible d'être labellisé ou protégé constitue le site unique de l'association gestionnaire, les implications pour cette dernière sont moins complexes que dans le cas d'une gestion multisite où il faut procéder à une gestion différenciée. C'est le cas de l'association versaillaise, qui gère trois sites :

- «Paul Philippe », un site urbain possédé en propre, financièrement bénéficiaire en raison de ses antécédents historiques,

- « La Ménagerie », un site rural en location au domaine national du Château de Versailles qui coûte beaucoup plus que le montant de ses cotisations,

27 - le «Clos Saint-Antoine », une aubaine urbaine récente de terrain prêté gracieusement par Versailles Habitat pour créer de nouveaux jardins familiaux mais qui s'avère un investissement ne pouvant s'amortir qu'en une dizaine d'années alors que sa convention d'occupation précaire n'est garantie que pour sept ans.

28 La labellisation ne doit donc pas seulement impliquer une exigence d'ouverture vers l'extérieur, elle devrait aussi générer une aide de l'extérieur. En particulier dans l'accompagnement des jardiniers car il est indispensable de faire vivre les jardins familiaux. Cela ne peut revenir aux administrateurs bénévoles dont la charge de la gestion au quotidien dépasse souvent déjà leurs possibilités et disponibilités. Cette reconnaissance n'est pas nouvelle :

Établir des règlements et des statuts est insuffisant. Il s'agit de les faire vivre par la participation des jardiniers et la transparence des décisions de gestion. Ces qualités d'animation se détectent, parfois parmi les jardiniers, mais elles existent souvent dans l'entourage des jardins au travers d'une association, d'un centre social, d'une mission locale... Peu importe qui anime, pourvu qu'il soit impliqué dans le projet commun et reconnu par l'ensemble des usagers du jardin comme le régulateur de la vie collective. L'animateur permet également une bonne communication avec les acteurs institutionnels ${ }^{5}$.

\section{En guise de conclusion : pas de protection des jardins familiaux sans accompagnement}

La présente contribution se veut avant tout être un retour d'expérience d'une labellisation d'un site de jardins familiaux, en particulier au niveau des implications de cette labellisation sur le vécu de l'association gérant ces jardins. Le développement et la 
pérennité des jardins familiaux sont intimement liés à la question de leur gestion comme l'attestent l'histoire et l'actualité de l'Association des jardins familiaux de Versailles et des communes environnantes gérant le site Paul Philippe labellisé « Jardin remarquable » en 2014. Reçue avec fierté et espoir, cette labellisation a certes eu des répercussions positives sur les jardins et sur certains jardiniers mais elle a aussi fait ressortir les faiblesses inhérentes à ce type d'institution: moyens financiers aléatoires, absence de compétences juridiques et administratives suffisantes, peu de disponibilités pour les ouvertures au public qui ont tendance à se multiplier, jardiniers familiaux peu enclins à s'ouvrir sur l'extérieur, essoufflement du bénévolat et risque d'effondrement par l'abandon des gestionnaires. Autant de carences qui menacent l'avenir des jardins familiaux tout autant que la pression immobilière. Protéger les jardins familiaux en les labellisant ou en les classant ne saurait donc suffire sans un accompagnement idoine des gestionnaires des associations loi de 1901 qui en ont la charge.

\section{NOTES}

1. - Pierre Desnos est traducteur retraité (Berlin, Paris, Versailles - Allemand et Anglais), ancien chargé d'enseignement à l'École supérieure d'interprètes et de traducteurs (université de ParisDauphine).

2. - Camille Boyer, curé de Saint-Symphorien. Voir le site: http:// www.academiedeversailles.com/_219624 [consulté le 11/10/2018].

3. - Extrait du courrier d'André Mignot, maire de Versailles, à l'Association des jardins familiaux, en date du 19 avril 1955 (ACV, 6 Z 23).

4. - Comme développé dans mon ouvrage : DESNOS, Pierre. Histoire des jardins ouvriers et familiaux de Versailles 1901-2011. Versailles : ASRIEUPE, août 2011, p. 83-84.

5. - Collectif [1999]. Guide méthodologique, les jardins familiaux, appropriation et intégration paysagère. [En ligne], consulté le 19 décembre 2017, [http://jardins-partages.org/telechargezmoi_files/ methodoJF.pdf].

\section{RÉSUMÉS}

Les jardins familiaux "pur jus », historiques, urbains et pleinement autonomes des Petits-Bois à Versailles ont reçu le label « Jardin remarquable » en 2014. La reconnaissance ne vaut cependant pas ipso facto pérennisation dès lors que la question de la gestion est négligée, ce qui est trop 
souvent le cas lorsque l'on aborde la question de la sauvegarde de ce type d'institution. Dans une première partie, nous donnerons le point de vue de l'historien. En déclinant l'histoire des jardins ouvriers et familiaux de Versailles et communes environnantes (1901-2017), cet article se propose avant tout d'en analyser le mode de fonctionnement du point de vue des gestionnaires (philanthropes, élus puis jardiniers). Nous verrons ensuite le point de vue d'un administrateur actuel. La labellisation du site Paul Philippe de Petits-Bois (Versailles) a confronté les administrateurs des jardins familiaux à des problématiques nouvelles: ouverture vers l'extérieur, remise en cause de pratiques anciennes, découverte des différences entre jardins familiaux urbains et ruraux, complexité d'une gestion difficilement maîtrisable par des bénévoles eux-mêmes jardiniers. Le développement et la pérennité des jardins familiaux sont intimement liés à la question de leur gestion comme l'attestent l'histoire et l'actualité de l'association versaillaise. Protéger les jardins familiaux en les labellisant ou en les classant ne saurait suffire si l'on ne trouve pas un mode d'accompagnement idoine des gestionnaires des associations loi de 1901 qui en ont la responsabilité.

In 2014 the historic and fully independent urban allotment gardens of Petits-Bois in Versailles, in their pure and traditional form, were given a French label for 'Remarkable Gardens', awarded by the Ministry of Culture. Unfortunately, this official recognition in itself does not necessarily guarantee their future if the question of the gardens' management is not properly addressed. This is all too often the case when it comes to dealing with the preservation of an institution of this type. In a first part of this article, we shall give the historian's point of view, recounting the history of allotment gardens in Versailles and its neighbouring municipalities between 1901 and 2017. We try to evaluate the way in which these gardens functioned from the point of view of those running them, such as philanthropists, elected municipal officials or the gardeners themselves. In a second part of the article, we shall examine the point of view of an administrator today. The 'Remarkable Garden' label awarded to the Paul Philippe site in PetitsBois gave the allotment administrators a whole new set of problems: the problem of opening up the garden to outside visitors, of reviewing longstanding practices and calling them into question, of understanding the differences between urban and rural allotment gardens. For today's volunteers-the gardeners themselves-it is sometimes difficult to master the complexities of these management problems. The development and survival of allotment gardens is intimately linked with this question of their management, as the history and current situation of the Versailles association shows. Preserving allotment gardens by giving them labels or statutory protection as historic monuments is not adequate. Appropriate ways of helping the garden's managers, operating under the terms of the French law of 1901 on non-profit associations, will also have to be found.

\section{INDEX}

Keywords : workers' allotment gardens, historic family gardens, Versailles, Petits-Bois, protection, 'Remarkable Garden' label, management, philanthropists, elected officials, gardeners, autonomy, volontary non-profit associations

Mots-clés : jardins ouvriers, jardins familiaux historiques, Versailles, Petits-Bois, protection, label « Jardin remarquable », gestion, gestionnaires philanthropes, élus et jardiniers, autonomie, bénévolat, mesures d'accompagnement 


\section{AUTEUR}

\section{PIERRE DESNOS}

Président fondateur de l'Association des riverains États-Pershing, président des Amis des forêts de Versailles et Fausses-Reposes, vice-président de l'Association des jardins familiaux de Versailles et communes environnantes pierre.desnos@wanadoo.fr 